\title{
Real-time Sensing of Trace Biomarkers from Viruses with a Microfluidic Immunosensor: A Case Study of SARS-CoV-2 Detection in Cold-chain Food
}

Haochen Qi†, Jian Zhang†, Jayne Wu*, Xin Fang, Fangshuo You, Wenci Zhao, Xiaoru Liu, Ying Chen, Lei Zheng*

H. Chen†, Dr. J. Zhang†, X. Fang, F. You, W. Zhao, X. Liu

School of Electronic Science and Applied Physics, Hefei University of Technology, Hefei 230009, China.

$\uparrow$ These authors contributed equally to this work.

Prof. J. Wu

Department of Electrical Engineering and Computer Science, the University of Tennessee, Knoxville, TN 37996, USA

E-mail: jaynewu@utk.edu

Prof. Y. Chen

Agro-product Safety Research Centre, Chinese Academy of Inspection and Quarantine, Beijing 100123, China.

Prof. L. Zheng

School of Food and Biological Engineering, Hefei University of Technology, Hefei 230009, China

E-mail: lzheng@hfut.edu.cn

Keywords: immunosensor, microfluidic enrichment, virus detection, tace biomarkers, SARS$\mathrm{CoV}-2$

Limited to low concentration of virions in food or environmental samples, real-time and direct detection of viruses without target amplification is hard to achieve. Utilizing an interdigitated microelectrode chip modified with an antibody probe, and integrating nanofluidic enrichment with interfacial capacitance sensing, an immunosensor is presented for detection of trace level spike-protein from SARS-CoV-2. It reaches a limit of detection low to $2.29 \times 10^{-6} \mathrm{ng} / \mathrm{mL}$ in 20 $\mathrm{s}$, with a wide linear range of $\left(10^{-5} \sim 10^{-1} \mathrm{ng} / \mathrm{mL}\right)$ and a high selectivity of $6.02 \times 10^{5}: 1$. The cost for single test can be controlled about 1 dollar. This sensor with its test strategy provides a competitive solution for real-time, sensitive, selective and large-scale application for virus detection in food and environment, and is demonstrated by SARS-CoV-2 detection in coldchain food. 


\section{Introduction}

The contamination of pathogens in food and environment has always been a serious problem worldwide for food safety, entry-exit quarantine, epidemic prevention and public health management. Although viral epidemics are less reported for food safety compared with bacteria $^{[1]}$, their transmissibility is not weak at all. More typical examples have been showing their destructive power, such as large outbreaks of hepatitis $\mathrm{A}^{[2,3]}$ and norovirus infection. ${ }^{[4,5]}$ As expected, these viruses are detected in various foods as well as waters, ${ }^{[1,6,7]}$ which exacerbates the spread of the disease. Therefore, the viral contamination detection in food poses a more great challenge in recent decade. ${ }^{[8]}$

Since winter 2019, the outbreak of a so-called coronavirus disease 19 (COVID-19) has become a global pandemic, with the culprit being a new SARS coronavirus (SARS-CoV-2). ${ }^{[9]}$ Although there is not yet a direct evidence to indicate the foodborne characteristic of SARSCoV-2, food contamination of SARS-CoV-2 may present a risk for virus spreading, which attracts considerable attention in many countries. ${ }^{[10-12]}$ SARS-CoV-2 virions adhering on solid surface are reported to be stable, with a viability up to longer than $72 \mathrm{~h}$ (on plastic). ${ }^{[13]}$ As a matter of fact, besides the wet markets providing a breeding ground for the virus, ${ }^{[14]}$ positive identification of SARS-CoV-2 on cold-chain foods are frequently reported in China recently, including the confirmed SARS-CoV-2 virions on plastic packing. Due to the below $0{ }^{\circ} \mathrm{C}$ temperature during the cold-chain transportation, the viruses can have a longer survival time than in room-temperature environment. On the other hand, the virus concentration may be of trace level, while a large number of samples needs to be tested. Therefore, sensitive, fast and low-cost SARS-CoV-2 detection is urgently needed for cold-chain foods.

For virus detection in food, there are mainly two types of techniques, culture/counting methods and polymerase chain reaction (PCR) based methods. ${ }^{[7,8]}$ Because the culture process is very time-consuming, PCR based methods have become a mainstream approach. ${ }^{[8,15]}$ Numerous works on various virus detection by PCR or reverse transcription (RT)-PCR in food 
have been reported, including hepatitis $\mathrm{A},{ }^{[15-17]}$ hepatitis $\mathrm{E},{ }^{[18]}$ norovirus. ${ }^{[15-17]}$ Although PCR techniques have been developed for more than 30 years, the operation is still complicated, and the required turnaround time cannot be shorter than several hours. ${ }^{[15]}$ Based on the good specificity and versatile sensing mechanisms, bio-probe based sensors for virus detection are becoming more practicable in recent years as promising approaches. ${ }^{[19-21]}$ Also due to the high specificity, reproducibility, and stability, antibody based detections of causative agent are continually adopted in food safety detection. ${ }^{[22,23]}$

Limited to the low concentration, direct detection of virus without complicated amplification is always hard to achieve. For SARS-CoV-2, the virion binds directly with its receptor through spike (S-) protein on its surface, and the subunit of S1 serves as the receptorbinding domain (RBD). ${ }^{[24-26]}$ As a result, S-protein (S1 subunit) is selected as a preferred biomarker for SARS-CoV-2 detection. ${ }^{[27-29]}$ Because the S-protein existing on the periphery of the virion is exposed on the food surface independent of body infection and viral assembly, at a quantity more than viral RNA itself, it can act as a good biomarker for food contamination of SARS-CoV-2. For specific recognition of certain antigen, antibody is considered as a promising probe due to the usually high affinity between antigen-antibody. ${ }^{[30]}$

Here, a sensor for trace S-protein (S1 subunit) detection associated with cold-chain food is developed for real-time SARS-CoV-2 contamination screening. An anti-SARS-CoV-2 Sprotein antibody is immobilized on a low-cost interdigitated microelectrode (IDME) chip. Then an alternating current (AC) signal is applied to the IDME to induce a dielectrophoresis force on the protein particles. Thus, the S-proteins are rapidly driven toward the IDME surface in the nanofluid and captured by the antibodies. Utilizing the electrical double layer capacitance at the IDME surface as an ultra-sensitive indicator, the limit of detection (LOD) reaches $10^{-6}$ $\mathrm{ng} / \mathrm{mL}$, while the time from sample to result is as short as $20 \mathrm{~s}$ due to effective target enrichment embedded into the detection process. Meanwhile, the detection is specific against the interferences, demonstrating a satisfactory selectivity in S-protein recognition. This report 
constructs a cost-effective immunosensor as well as a simple detection strategy for real-time, selective, and large-scale screening of virus through its trace biomarkers.

\section{Result and Discussion}

\subsection{Characterization of Sensor Preparation}

For sensor development, the functionalization effect should be verified before its application. Aluminium (Al) deposited on the substrate is first verified for $\mathrm{Al}(\mathrm{A} 12 \mathrm{p})$ is dominant at the IDME surface before functionalization (Figure 1a), but it is difficult to found after the functionalization (Figure 1b). According to original dada, the peak area of Al2p decreases from 17544 to $1916 \mathrm{cps} \cdot \mathrm{eV}$ due to good antibody coverage on IDME surface, which is also confirmed by the appearance of N1s characteristic peak (Figure 1c). Rather than oxygen (O) and carbon (C), the element of nitrogen (N1s) as a characteristic element should be from the antibody. According to peak-differentiating and imitating (Figure 1d), the formula of N1s contains $(\mathrm{H} 2 \mathrm{~N}-\mathrm{C})$ and $(\mathrm{C}=\mathrm{N}),{ }^{[31]}$ which are exactly abundant in antibody. Sodium $(\mathrm{Na} 1 \mathrm{~s})$ and phosphorus (P2p) (Figure 1b) are accordant with the characteristic peaks from $\mathrm{Na}_{2} \mathrm{HPO}_{4}$ and $\mathrm{NaCl}$, the components of PBS solvent. The presence of P2p at the bare IDME (Figure 1a) could be residues of phosphoric acid from Al electrode etching during chip fabrication. In conclusion, the XPS spectrums validate a successful surface functionalization by antibody.

a

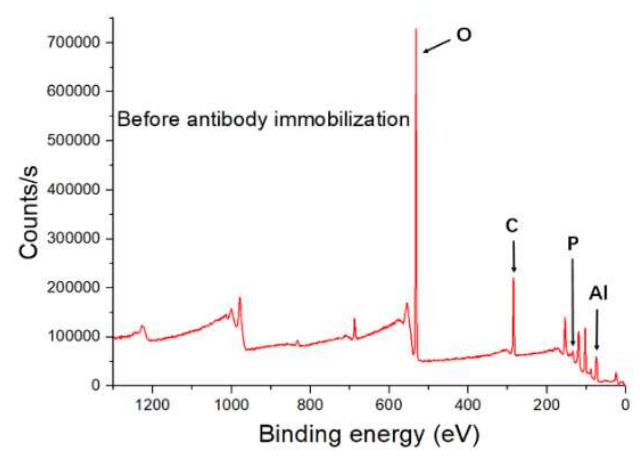

b

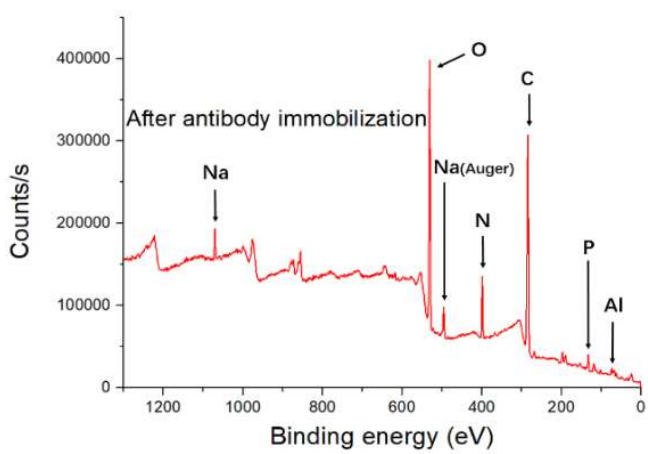



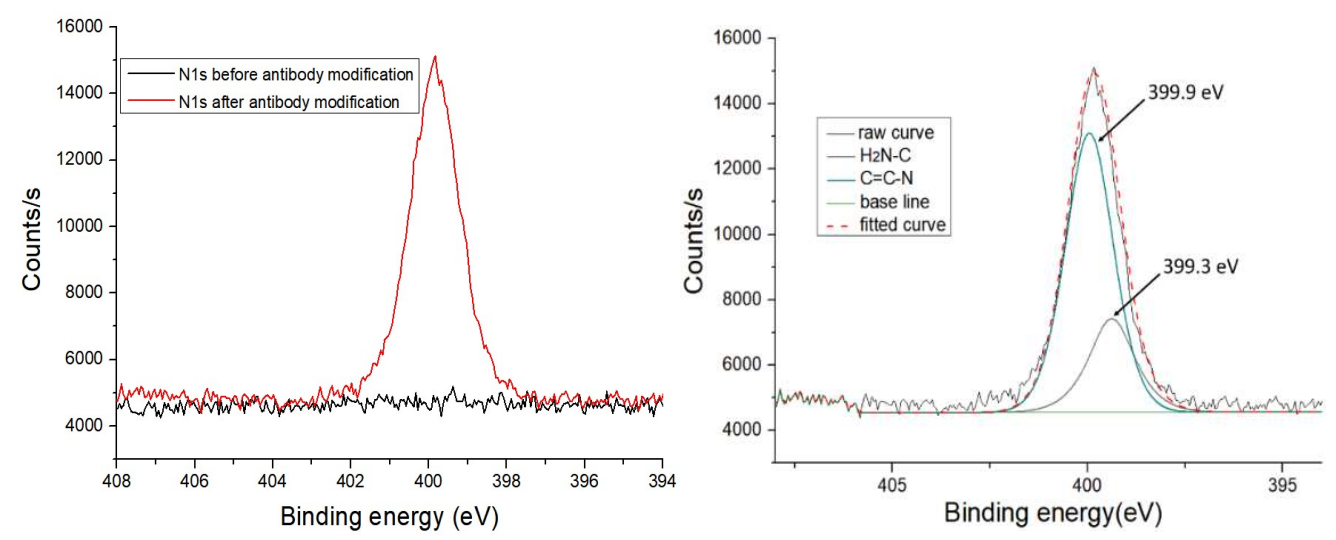

Figure 1. Full spectrum of XPS on a) thoroughly cleaned IDME surface before antibody modification and b) functionalized IDME surface after antibody modification. c) High resolution spectrums of nitrogen (N1s) before and after IDME modification. d) The peakdifferentiating and imitating of N1s.

In addition, Bode plot of impedance and phase angle from $10^{2}$ to $10^{5} \mathrm{~Hz}$ is acquired to reflect the change on the IDME surface during functionalization by electrical method. The impedance distinctly increases after antibody modification, but changes little after blocking (Figure 2a). Because the covered antibody layer makes the dielectric layer thicker on the IDME surface, causing a smaller conductivity than that of single electric double layer (EDL), the increase of impedance is easy to understand. The reason why the lactalbumin blocker changes little of the impedance is the smaller lactalbumin not making the dielectric layer thicker anymore. In the phase angle spectrum (Figure 2b), the lag of phase angle after antibody modification verifies the interfacial capacitance. Also, the blocking has little effect on the phase angle curve because of its weak influence on the capacitance. 


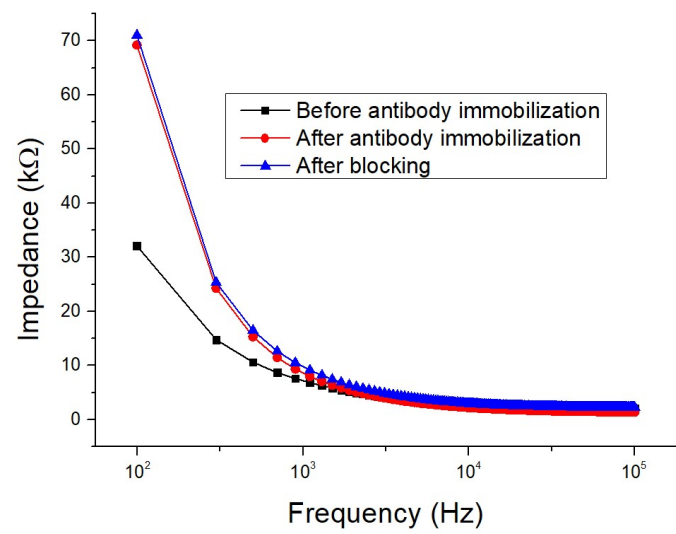

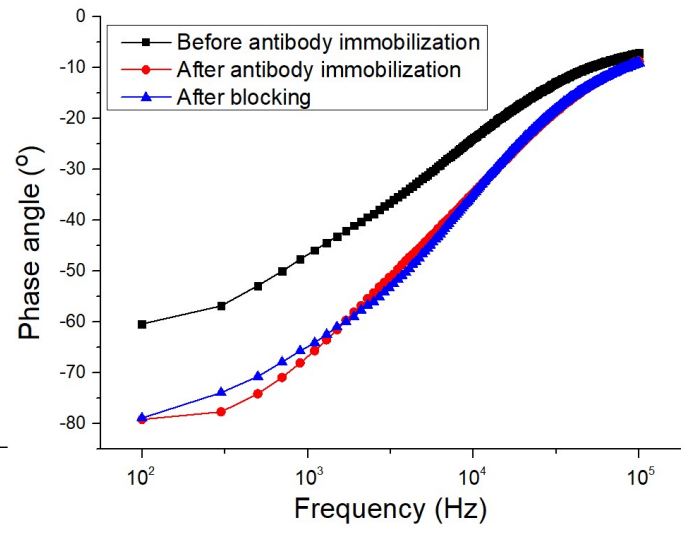

Figure 2. Bode plot of a) impedance and b) phase angle from $10^{2}$ to $10^{5} \mathrm{~Hz}$ as an electrical characterization for the IDME surface modification.

\subsection{Dose Response from S-protein in PBS Buffer}

The calibration for the IDME immunosensor should be first performed before its application. The S-protein is tested with tenfold increase from $10^{-5}$ to $10^{-1} \mathrm{ng} / \mathrm{mL}$ in $0.1 \times \mathrm{PBS}$ solution. Here the upper-limit concentration of $10^{-1} \mathrm{ng} / \mathrm{mL}$ is determined by observing the saturation phenomenon ${ }^{[32,33]}$ at $1 \mathrm{ng} / \mathrm{mL}$ (illustrated in Figure S1a, b in supporting information).

The typical transient curves of normalized capacitance vs. time are acquired from the sensors (Figure 3a). The curves can be clearly differentiated between different target concentrations, and exhibit increasingly downward slopes with the increase of S-protein concentration in $0.1 \times \mathrm{PBS}$. This indicates the sensor's primary performance of output monotonicity. The linearity of the transient curves become worse after $10 \mathrm{~s}$ when the concentration is high. Therefore, the response time of this sensor is chosen to be $20 \mathrm{~s}$.

For calibration using dose response (Figure 3b), the response of capacitance is normalized by the initial one, and $\mathrm{dC} / \mathrm{dt}$ represents the change of normalized capacitance in 1 minute's duration, which is found by least squares linear regression method. The calibration curve, Y $(\%)=-2.66 \lg X(\mathrm{ng} / \mathrm{mL})$, demonstrates an excellent semi-log linear relationship between the 
target concentration and the response from $10^{-5} \mathrm{ng} / \mathrm{mL}$ until $10^{-1} \mathrm{ng} / \mathrm{mL}$, with a correlation coefficient $\left(\mathrm{R}^{2}\right)$ of 0.994 . With the cut-off line $(\mathrm{Y}=2.05 \%)$ defined as a response value three standard deviations from the background, the LOD is calculated to be $2.29 \times 10^{-6} \mathrm{ng} / \mathrm{mL}$ plugging the cut-off value into the calibration equation. This LOD is at an ultra-low level. The dose response as well as the calibration demonstrates a quantitative performance of the sensor, with a wide linear range.

a

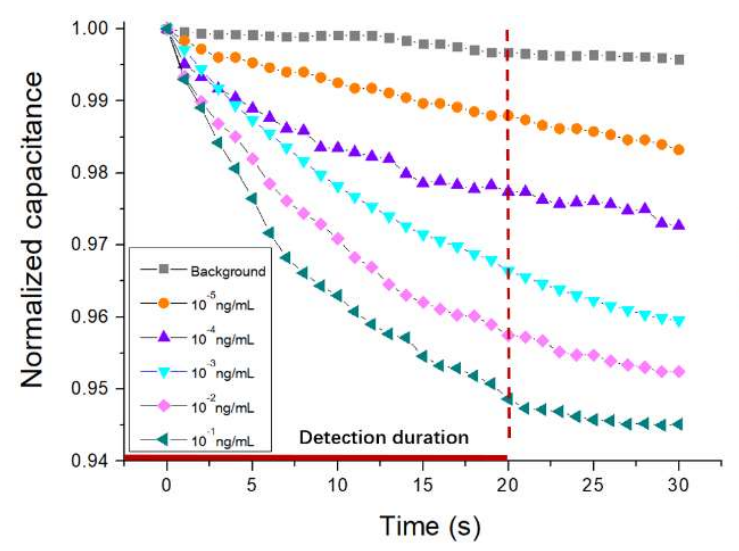

b

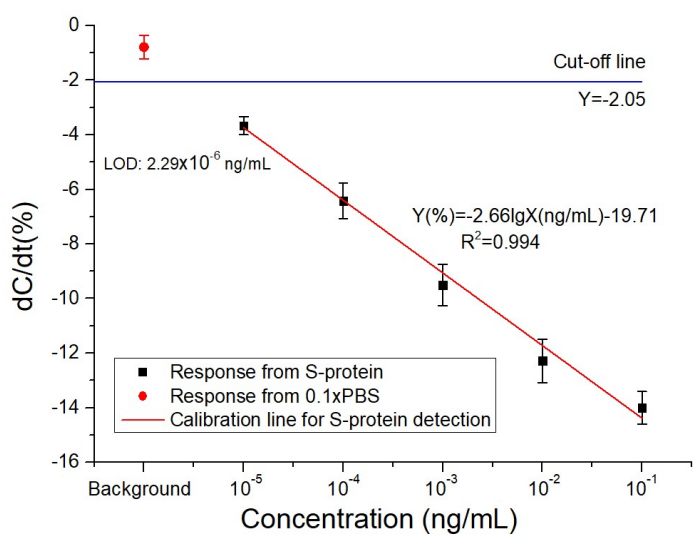

Figure 3. Response acquired from the immunosenors. a) The typical transient capacitance normalized by its initial value changing with time. Five concentrations of S-protein (from $10^{-5}$ to $10^{-1} \mathrm{ng} / \mathrm{mL}$ ) as well as the background of $0.1 \times$ PBS are continuously detected for $30 \mathrm{s.} \mathrm{b}$ ) Dose response and its calibration. The triplicate data are acquired from three freshly prepared sensors, and the error bar represents the standard deviation.

\subsection{Selectivity of S-protein against the Interferences}

For a qualified sensor, selectivity for the target is the most important performance. In this work, the acquired effective response is verified in three ways: (1) comparing the response from functionalized sensors with that from dummy sensors when S-protein is test, (2) comparing the response from S-protein with that from the background of $0.1 \times \mathrm{PBS}$, and (3) comparing the 
response from S-protein with that from the interferences. Here the dummy sensor is defined for an IDME chip without antibody functionalization to verify the probe's effectiveness.

According to the comparison result (Figure 4a), the response from background is within $1 \%$, the dummy sensors keep unresponsive, and the three interference molecules of SARSCoV-2 nucleocapsid (N-) protein, peptidoglycan (PGN), and lipopolysaccharide (LPS) all cause unobvious response. The biggest non-target response is from the N-protein of SARSCoV-2 especially when its concentration is high. The maximum of $-4.04 \%$ from N-protein at $10^{-1} \mathrm{ng} / \mathrm{mL}$ is equivalent to the response from S-protein at $4.27 \times 10^{-4} \mathrm{ng} / \mathrm{mL}$ calculated using the calibration line, then the selectivity ratio of this sensor is calculated to be $234: 1\left(10^{-1} \mathrm{ng} / \mathrm{mL}\right.$ : $4.27 \times 10^{-4} \mathrm{ng} / \mathrm{mL}$ ). Because N-protein is also a valuable marker for SARS-CoV-2 recognition, a weak response can help recognize the target in deed. Then, the real selectivity ratio should be obtained with PGN response, which is most significant (-1.68\%) except N-protein also at $10^{-1}$ $\mathrm{ng} / \mathrm{mL}$. Using the similar method, the selectivity ratio is calculated to be about $6.02 \times 10^{5}: 1$.

To demonstrate the capability of target recognition in hybrid matrices, S-protein detection is also performed in $0.1 \times$ PBS containing peptidoglycan $(\mathrm{PGN})$ and lipopolysaccharide (LPS) both of $10^{-3} \mathrm{mg} / \mathrm{mL}$, which most possibly exist at the food surface dissociated from various bacteria. In this test, the spiked S-protein is of $10^{-4} \mathrm{ng} / \mathrm{mL}$. Although the concentrations of nontarget molecules are 10 fold higher than that of S-protein, the response is only a bit larger than that in PBS without impurities (Figure 4b), and still within an acceptable tolerance from the calibration curve. The response is about $107.94 \%$ of the calibrated concentration, meeting the requirement of practical quantitative detection in hybrid matrices. 

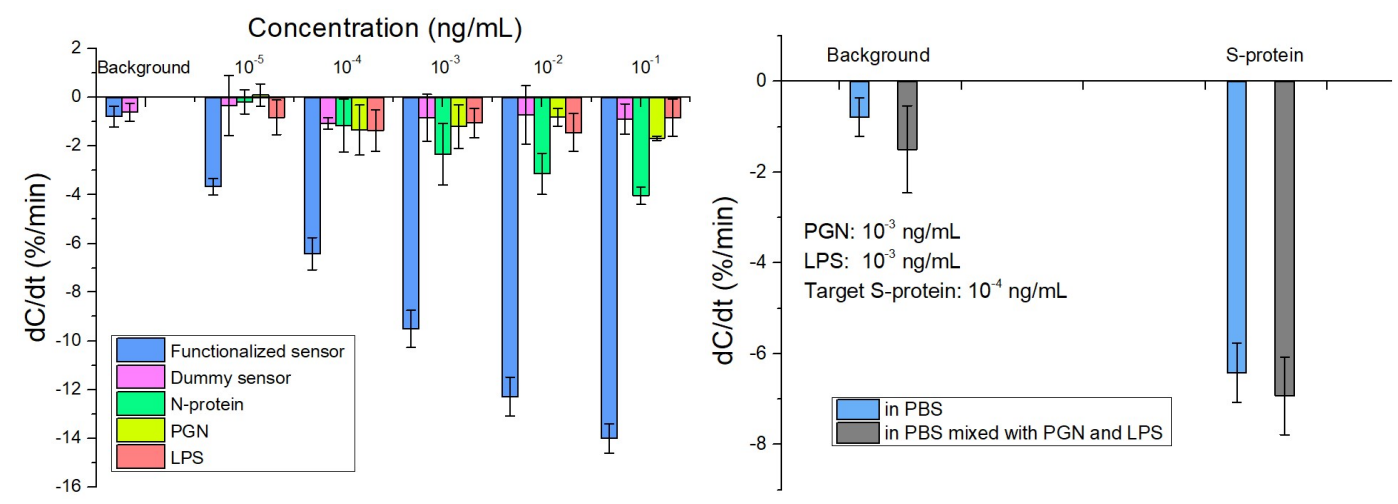

Figure 4. Specificity verification of the immunosenors. a) Specificity verification using different types of sensors and different analytes. The background is first tested to verify the blank control and the sensor blocking effect. Functionalized sensors are compared with dummy sensors (also blocked with lactalbumin), to characterize the antibody functionalization. The control group contains nucleocapsid (N-) protein, peptidoglycan (PGN) and lipopolysaccharide (LPS). b) Specificity verification in hybrid medium. A hybrid medium is constructed by mixing PGN and LPS in $0.1 \times$ PBS solution. The PGN and LPS concentrations in $0.1 \times$ PBS are both $10^{-}$ ${ }^{3} \mathrm{ng} / \mathrm{mL}$, and the spiked S-protein is at $10^{-4} \mathrm{ng} / \mathrm{mL}$.

\subsection{Detection of S-protein in Practical Media}

After verification of the sensor performance as well as the test strategy, we have applied this approach in practical media for trace S-protein detection. Three types of media associated with cold-chain food are modeled: melted tap water from the ice for cold-chain transportation, extracts from different fresh seafood surfaces, and soaking liquid from a packing bag for frozen food. Because the conformed food samples contaminated by SARS-CoV-2 are extremely difficult to obtain, different matrices spiked with S-protein are detected.

When S-protein detection in the tap water is performed (Figure 5a), the spiked concentration is from $10^{-5}$ to $10^{-1} \mathrm{ng} / \mathrm{mL}$. For comparison, the detection result from spiked $0.1 \times \mathrm{PBS}$ is provided together and the response from the tap water is lower than from PBS. The 
background of tap water is above zero, which may be caused by very few large particles such as bacteria and dust in the solution. If the adsorbed particles are too few in number and the particle size is large (up to $\mathrm{nm}$ or $\mu \mathrm{m}$ ), the interfacial capacitance will increase as illustrated in supporting information (Figure S2). In Figure 5a, although the baseline of the tap water is above zero, the variation tendency of response keeps a good agreement with that from spiked PBS, showing an expected dose relationship. For practical application, the response will reflect the real S-protein concentration after a simple baseline calibration.

a

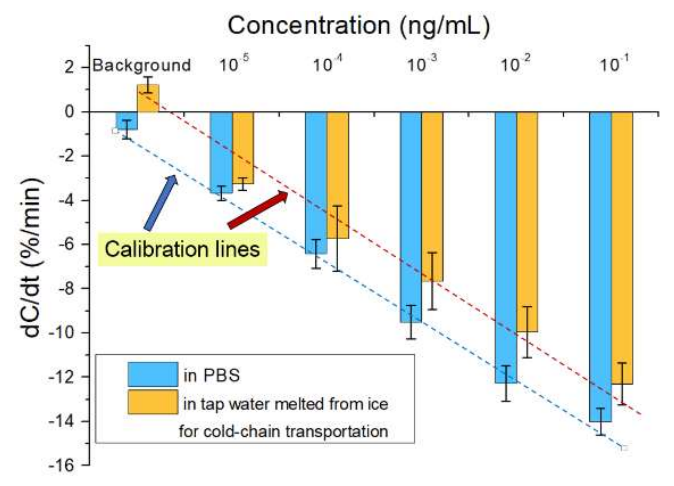

b

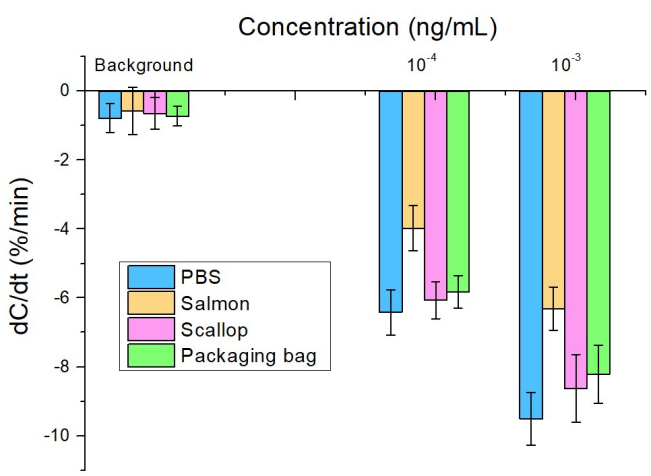

Figure 5. S-protein detection in practical samples. a) Dose response from S-protein in melted tap water from the ice for cold-chain transportation. 5 concentrations from $10^{-5}$ to $10^{-1} \mathrm{ng} / \mathrm{mL}$ are spiked and detected in the tap water, and the calibration is performed as a red dash line. The blue dash line as a standard calibration in PBS is provided for comparison. b) S-protein detection in 3 samples associated with cold-chain food. Leaching solutions are obtained from salmon, scallop and packing bag for beef, all having two theoretical concentrations $\left(10^{-4}\right.$ and $10^{-3} \mathrm{ng} / \mathrm{mL}$ ). The corresponding backgrounds are as a quality control for the sensors before application. The response in $0.1 \times \mathrm{PBS}$ is provided as a reference. 
Other detections for S-protein associated with cold-chain food are also performed with three types of materials, i.e., salmon, scallop and a packing bag for frozen beef (Figure $5 b$ ). Here two concentrations of $10^{-4}$ and $10^{-3} \mathrm{ng} / \mathrm{mL}$ are obtained by spiking, incubation and dilution steps as introduced in the section of methods. To get rid of the impurity such as lipid particles, centrifugation is also executed before final detection. Four backgrounds all show negligible response around $1 \%$ of $\mathrm{dC} / \mathrm{dt}$, indicating a qualified sensor preparation to block nonspecific adsorption. The target S-protein in various matrices can be distinctly recognized at both two concentrations, although the responses are smaller than that from PBS solution. In these results, the smallest response is from the salmon. Because the salmon sample has been processed in strips, and the lipid is rich at the surface, there are many organic molecules and particles dispersed and suspended in the turbid solution. Although high-speed centrifugation has made the background unresponsive, the positive response may be inhibited in some degree. For further practical application with these complex samples, a new calibration can be made for accurate quantitative determination of SARS-CoV-2 contaminant. By contrast, surface of the scallop and packing bag is much more smooth and dense, so that the interference is much less, and the S-protein can be more completely dispersed in PBS solution. As a result, the response from these two samples are more notable.

According to these results, S-protein in different cold-chain food samples can be successfully detected by our sensor and the test method.

\section{Conclusion}

In this work, a strategy for real-time, selective, and low-cost detection of S-protein from SARS-CoV-2 is presented based on a low-cost commercial IDME based sensor combined with interfacial capacitance sensing method. An anti-SARS-CoV-2 S-protein antibody is employed as a bio-probe immobilized on the IDME surface to specifically recognize the trace S-protein in cold-chain food associated samples. 
Due to effective utilization of DEP force, S-protein particles are attracted towards the sensor's surface and captured by the antibodies in $20 \mathrm{~s}$. Also, the response acquisition is completely integrated into the target enrichment process, thus the response time of $20 \mathrm{~s}$ can meet the real-time detection demand on site. Besides, the LOD of this detection reaches an ultra-low level, i.e., $10^{-6} \mathrm{ng} / \mathrm{mL}$. Meanwhile, the linear range of $\left(10^{-5} \sim 10^{-1} \mathrm{ng} / \mathrm{mL}\right)$ is extremely wide covering the possible range of S-protein concentration at the food surface. Even when testing a food sample with complex surface such as salmon strip, the presence and concentration of S-protein can be easily determined. In fact, cold-chain foods are mostly contaminated with SARS-CoV-2 virions by the workers carrying virus in food-processing factories. Because virus replication cannot occur at the food surface, the level of virus concentration should be very low, which poses a great challenge to traditional detection method. As such, the ultra-low LOD of the developed strategy shows an immense advantage in trace virus marker detection.

Another merit of this strategy is the high specificity. The selectivity for S-protein against the interference is calculated to be $6.02 \times 10^{5}: 1$, which means that the response from non-target molecules is equal to that from the target when their concentration ratio reaches $10^{5}$. Not only common organic molecules, but also various matrices as backgrounds are verified to be nonresponsive, with known or unknown components. Therefore, the sensor has demonstrated the required specificity to identify the S-proteins from practical samples with complex backgrounds. The cost for a single sensor device is estimated to be about 1 dollar. As a result, this sensor is designed to be a disposable chip. Meanwhile, the impedance analyzer for capacitance detection is also inexpensive and lightweight. As discussed previously, the detection operation as well as the pretreatment is simple only including dilution and centrifugation steps. Therefore, this sensor can be operated by a lay person. Based on the above characteristics, the platform and method for direct biomarker instead of virus (or RNA) detection provides a promising solution for large-scale applications in food and environment monitoring requiring quick response, low LOD, high specificity, user-friendly operation and low cost as well. 


\section{Experimental Section}

Target Enrichment and Sensing Mechanism: For trace particle detection in liquid, the preconcentration of target particles is always crucial to a successful test. ${ }^{[34-36]}$ Compared with most techniques for pre-concentration needing an extra incubation or equipment, $\mathrm{AC}$ electrokinetic (ACEK) effects can manipulate nanoparticles efficiently to realize target enrichment rapidly without extra processes or devices. ${ }^{[37-39]}$ For relatively large molecules such as proteins, dielectrophoresis (DEP) force, an important ACEK effect, has been demonstrated as a dominant force applied on the particles. ${ }^{[40,41]}$ In this work, an IDME chip is used as a physical device to induce DEP effect. DEP force can be expressed as a function of electric field (Equation S1 in the supporting information), affected by the electric field gradients in the liquid. The voltage applied to the IDME has a positive correlation to the DEP force. To illustrate the space distributions of electric potential and the induced DEP in solution, their simulation results are provided (in Figure S3), according to which the potential and velocity field are described under the test condition, and the directional movement of S-proteins towards the IDME surface is simulated. Therefore, the nanofluidic manipulation and enrichment of S-protein can be expected.

When an electrode is immersed in a solution, electric double layer (EDL) will appear due to the accumulated charges at the electrode surface and the layer of induced counter ions above the surface. ${ }^{[2,43]}$ The layer of antibody and lactoalbumin molecules at the IDME surface plus the EDL forms the initial dielectric layer of the interfacial capacitance (as shown in Figure S4). When S-proteins are captured by the antibodies, the dielectric layer becomes thicker, and as a result, the interfacial capacitance becomes smaller (as deduced in Equation S2). The change of normalized capacitance per minute, i.e., $\mathrm{dC} / \mathrm{dt}(\% / \mathrm{min})$, directly reflects the S-protein adsorption level, by which the S-protein concentration in solution can be indicated. Using an IDME of micron scale, $\mathrm{dC} / \mathrm{dt}$ is a competitive parameter to reflect tiny change at the electrode interface. ${ }^{[38,44]}$ Therefore, ultra-trace S-protein detection can be expected. In practice, the lab- 
prepared sensors may have inconsistency due to different effective electrode surface and total recognition sites. Here, the normalization by initial capacitance (as described in Equation S2) has no relation with the initial electrode surface, thus can minimize the deviation between the response from different sensors.

Materials and Reagents: The S-protein (S1 subunit) is purchased from Cellregen (Beijing) Life Science and Technology Co., LTD, which is recombinant and expressed by prokaryotic system with the host of Escherichia coli. The molecular weight this protein is $75.3 \mathrm{kDa}$, with a purity above $90 \%$. The sodium dodecyl sulfate polyacrylamide gel electrophoresis (SDS-PAGE) result is provided in Figure S5a, and the amino acid sequence is shown in Figure S5b. The antiSARS-CoV-2 spike protein S1 (mouse monoclonal IgG) is purchased from Anygo Technology Co., LTD, China, with a purity above $95 \%$. The recombinant N-protein is also provided by Cellregen (Beijing) Life Science and Technology Co., LTD, and the LPS (L2880) is ordered from Sigma-Aldrich Co., LTD. The PGN is bought from Nanjing Duly Biotechnology Co. LTD, China. The IDME chips are modified based on commercially available surface acoustic wave (SAW) chips (AVX Corps' KYOCERA 433K).

Sensor Preparation and Test Protocol: The SAW chip is packaged by a ceramics chamber with the outer size of $5 \times 3.5 \mathrm{~mm}$ (as shown in Figure S6a). The chamber is enclosed by a metal cover. Inside the chamber, an aluminum IDME structure is fabricated on a ceramic substrate (as shown in Figure S6b), the widths of finger and gap of the IDME are $2 \mu \mathrm{m}$ and $1.5 \mu \mathrm{m}$, respectively. For sensor preparation, the metal cover is first removed, and the chip is thoroughly cleaned by soaking in acetone for $25 \mathrm{~min}$, soaking in isopropyl alcohol for $2 \mathrm{~min}$ and rinsing with purified water for $10 \mathrm{~s}$. Then, the chip is treated with ozone for $30 \mathrm{~min}$ to improve the hydrophilia of the electrode surface. After that, $10 \mu \mathrm{L}$ antibody ( $10 \mu \mathrm{g} / \mathrm{mL}$ in $0.05 \times \mathrm{PBS})$ is added into the chamber and incubated for $24 \mathrm{~h}$ in a humidor. The final step for is blocking for $3.5 \mathrm{~h}$ using lactoalbumin $(100 \mu \mathrm{g} / \mathrm{mL}$ in $0.05 \times \mathrm{PBS})$ to cover the unfunctionalized IDME area. 
The functionalized sensor is first connected to an impedance analyzer (TH2829C, Tonghui Electronic Co. LTD) (as shown in Figure S7a). After the sample is dropped into the sensor's chamber, an AC signal is applied for a selected duration of $20 \mathrm{~s}$.

The test procedures mainly include incubation, extraction and centrifugation steps (as shown in Figure S7b). Before measurement, all the practical samples should be pretreated. The conductivity of raw tap water (melted from the ice for cold-chain transportation) is tested to be $0.012 \mathrm{~S} / \mathrm{m}$. Then the raw tap water is $1: 1$ mixed with $0.19 \times \mathrm{PBS}$ to obtain a mixture with the conductivity of $0.141 \mathrm{~S} / \mathrm{m}$, which is as same as that of $0.1 \times$ PBS. During all the detection in this work, the solution conductivity is kept to be $0.141 \mathrm{~S} / \mathrm{m}$, which makes an equal effect on DEP by solution conductivity. ${ }^{28,29}$ Then S-protein is added in this mixed background also called as "tap water". For cold-chain-food associated samples (salmon, scallop and a packing bag for frozen beef), a small piece of certain sample about $8 \times 8 \mathrm{~mm}$ is first cut off, and $10 \mu \mathrm{L}$ S-protein of $1 \mathrm{ng} / \mathrm{mL}$ is spread over it. After the solid sample is incubated for $12 \mathrm{~h}$ in a closed tube at $4{ }^{\circ} \mathrm{C}$, $990 \mu \mathrm{L} 0.1 \times \mathrm{PBS}$ is added into the tube. Then the solution is sufficiently agitated and centrifuged at $3000 \mathrm{rpm}$ for $10 \mathrm{~min}$. The supernatant from this mixture with a theoretical S-protein concentration of $10^{-2} \mathrm{ng} / \mathrm{mL}$ is ready for further dilution to obtain the final samples with theoretical concentrations of $10^{-3}$ and $10^{-4} \mathrm{ng} / \mathrm{mL}$. During the pretreatment for salmon slice, an additional centrifugation at $5000 \mathrm{rpm}$ for $10 \mathrm{~min}$ is necessary to remove the abundant interference, and the floating lipid layer should be excluded when the supernatant is collected. To obtain the corresponding backgrounds for these practical samples, similar process is performed except for the S-protein spreading and incubation steps.

The frequency of the AC signal is set to be $100 \mathrm{kHz}$ according to previous study, ${ }^{28,41}$ and the voltage is optimized to be $100 \mathrm{mV}$ according to the optimization (described in Figure S8). Based on the measured capacitance, $\mathrm{dC} / \mathrm{dt}$ can be calculated and analyzed. To demonstrate the sensor's repeatability and consistency, all the data are presented with error bars, which represent the standard deviation obtained from three sensors of the same batch. 


\section{Supporting Information}

Supporting Information is available from the Wiley Online Library or from the author.

\section{Acknowledgements}

This work was supported by the National Key R\&D Program of China (2016YFD0401104, 2017YFC1600603, 2019YFC1605302), National Natural Science Foundation of China (62074047), Anhui Provincial Natural Science Foundation of China (1908085MF180), and the open research fund of the Key Laboratory of MEMS of the Ministry of Education, Southeast University, China. J. Wu thanks the support from the University of Tennessee Initiative of PON/POC Nanobiosensing, Institute of Secure and Sustainable Environment and USDA NIFA (Grant No. 2017-67007-26150).

\section{Conflict of Interest}

The authors declare no conflict of interest.

Received: ((will be filled in by the editorial staff))

Revised: ((will be filled in by the editorial staff)) Published online: ((will be filled in by the editorial staff))

\section{References}

[1] C. Bachofen, Curr. Clin. Micro. Rpt. 2018, 5, 143.

[2] E. Severi, L. Verhoef, L. Thornton, B. Guzman-Herrador, M. Faber, L. Sundqvist, R. Rimhanen-Finne, A. M. Roque-Afonso, S. L. Ngui, F. Allerberger, A. Baumann-Popczyk, L. Muller, K. Parmakova, V. Alfonsi, L. Tavoschi, H. Vennema, M. Fitzgerald, M. Myrmel, M. Gertler, J. Ederth, M. Kontio, C. Vanbockstael, S. Manda, M. SadkowskaTodys, M. E. Tosti, B. Schimmer, J. O’Gorman, K. Stene-Johansen, J. J. Wenzel, G. Jones, K. Balogun, A. R. Ciccaglione, L. O’Connor, L. Vold, J. Takkinen, C. Rizzo, Euro Surveill. 2015, 20, 21192.

[3] K. Y. Yoon, B. C. Chun, H. K. Lee, Y. S. Seo, J. H. Shin, Y. S. Hong, H.S. Sim, J.Y. Kim, J.Y. Kim, Y.S. Park, D. W. Park, J.W. Sohn, M. J. Kim, J. Clin. Virol. 2009, 46, 184.

[4] A. J. Hall, J. Infect. Dis. 2012, 205, 1622.

[5] Y. Somura, M. Nagano, K. Kimoto, M. Oda, K. Mori, T. Shinkai, K. Sadamasu, Lett. Appl. Microbiol. 2019, 69, 175. 
[6] H. A. Alhadrami, S. Al-Amer, Y. Aloraij, F. Alhamlan, R. Chinnappan, K. M. Abu-Salah, S. Almatrrouk, M. M. Zourob, ACS Omega 2020, 5, 12162.

[7] A. Bosch, G. Sánchez, M. Abbaszadegan, A. Carducci, S. Guix, F. S. Le Guyader, R. Netshikweta, R. M. Pintó, W. H. M. van der Poel, S. Rutjes, D. Sano, M. B. Taylor, W. B. van Zyl, D. Rodríguez-Lázaro, K. Kovač, J. Sellwood, Food Anal. Methods 2011, 4, 4.

[8] P. Rajapaksha, A. Elbourne, S. Gangadoo, R. Brown, D. Cozzolino, J. Chapman, Analyst 2019, 144, 396.

[9] T. P. Velavan, C.G. Meyer, Trop. Med. Int. Health 2020, 25, 278.

[10] Z. Ceylan, R. Meral, T. Cetinkaya, VirusDis. 2020, 31, 154.

[11] Y. H. Roos, Food Eng. Rev. 2020, 12, 251.

[12] A. Aiyar, P. Pingali, Food Secur. 2020, 3, 749.

[13] N. V. Doremalen, T. Bushmaker, D. H. Morris, M. G. Holbrook, V. J. Munster, New Engl. J. Med. 2020, 382, 1564.

[14] Nat. Food 2020, 1, 241. https://doi.org/10.1038/s43016-020-0090-1

[15] S. M. Rajiuddin, T. Jensen, T. B. Hansen, A. C. Schultz, Food Environ. Virol. 2020, 12, 226.

[16] J. Chen, X. Wu, G. Sanchez, W. Randazzo, Food Control 2019, 107, 106818.

[17] C. Hennechart-Collette, F. Niveau, S. Martin-Latil, A. Fraisse, S. Perelle, Int. J. Food Microbiol. 2019, 311, 108349.

[18] I. L. A. Boxman, C. C.C. Jansen, A. J. T. Zwartkruis-Nahuis, G. Hägele, N. P. Sosef, R. A. M. Dirks, Int. J. Food Microbiol. 2020, 333, 108791.

[19] J. Zhuang, J. Yin, S. Lv, B. Wang, Y. Mu, Biosens. Bioelectron. 2020, 163, 112291.

[20] C. Cheng, H. Cui, J. Wu, S. Eda, Microchim. Acta 2017, 184, 1649.

[21] C. Cheng, J. Wu, E. Fikrig, P. Wang, J. Chen, S. Eda, P. Terry, ChemElectroChem 2017, 4,485 .

[22] H. Jayan, H. Pu, D. W. Sun, Trends Food Sci. Tech. 2020, 95, 233. 
[23] J. Zhang, Y. Jiang, X. Xia, J. Wu, R. Almeida, S. Eda, H. Qi, Biosens. Bioelectron. 2020, $165,112366$.

[24] D. Wrapp, N. Wang, K. S. Corbett, J. A. Goldsmith, C. L. Hsieh, O. Abiona, B. S. Graham, J. S. McLellan, Science 2020, 367, 1260.

[25] A. C. Walls, Y. J. Park, M. A. Tortorici, A. Wall, A. T. McGuire, D.Veesler, Cell 2020, $180,281$.

[26] X. Ou, Y. Liu, X. Lei, P. Li, D. Mi, L. Ren, L. Guo, R. Guo, T. Chen, J. Hu, Z. Xiang, Z. Mu, X. Chen, J. Chen, K. Hu, Q. Jin, J. Wang, Z. Qian, Nat. Commun. 2020, 11, 1620.

[27] X. Zhang, Q. Qi, Q. Jing, S. Ao, Z. Zhang, M. Ding, M. Wu, K. Liu, W. Wang, Y. Ling, Z. Zhang, W. Fu, 2020, arXiv: 2003.12529v1

[28] S. Mavrikou, G. Moschopoulou, V. Tsekouras, S. Kintzios, Sensors 2020, 20, 3121.

[29] A. Ahmadivand, B. Gerislioglu, Z. Ramezani, A. Kaushik, P. Manickam, S. A. Ghoreishi, 2020, arXiv: 2006.08536

[30] R. Chen, X. Huang, H. Xu, Y. Xiong, Y. Li, ACS Appl Mater Inter. 2015, 7, 28632.

[31] A. K. S. Ang, E. T. Kang, K. G. Neoh, K. L. Tan, T. B. Lim, Polymer 2000, 41, 489.

[32] H. Qi, M. Zhao, H. Liang, J. Wu, Z. Huang, A. Hu, J. Wang, Y. Lu, J. Zhang, Electrophoresis 2019, 40, 2699.

[33] J. Zhang, X. Fang, J. Wu, Z. Hu, Y. Jiang, H. Qi, L. Zheng, X. Xuan, Biosens. Bioelectron. 2020, 150, 111879.

[34] M. S. Myint, Y. J. Johnson, N. L. Tablante, R. A. Heckert, Food Microbiol. 2006, 23, 599.

[35] N. V. Nguyen, C. P. Jen, Biosens. Bioelectron. 2018, 121, 10.

[36] Q. Wang, T. R. Slaney, W. Wu, R. Ludwig, L. Tao, A. Leone, Anal. Chem. 2020, 92, 10327

[37] H. Cui, C. Cheng, X. Lin, J. Wu, J. Chen, S. Eda, Q. Yuan, Sensor. Actuat. B-Chem. 2016, $226,245$. 
[38] J. Zhang, J. Wang, J. Wu, H. Qi, C. Wang, X. Fang, C. Cheng, W. Yang, Microfluid. Nanofluid. 2019, 23, 2.

(39) C. S. Ivanoff, J. Wu, H. Mirzajani, C. Cheng, Q. Yuan, S. Kevorkyan, R. Gaydarova, D. Tomlekova, Biomed. Microdevices. 2016, 18, 84.

(40) X. Liu, C. Cheng, J. Wu, S. Eda, Y.Guo, Biosens. Bioelectron. 2016, 90, 83.

(41) C. Cheng, J. Wu, J. Chen, Electrophoresis 2018, 40, 890.

(42) H. Morgan, N. G. Green, Research Studies Press. 2002.

(43) R. Oueslati, C. Cheng, J. Wu, J. Chen, Biosens Bioelectron. 2018, 108, 103.

(44) J. Zhang, R. Oueslati, C. Cheng, L. Zhao, J. Chen, R. Almeida, J. Wu, Biosens. Bioelectron. 2018, 112,48 . 